\title{
Study on Fat and Thin Analysis of Tongue Body Based on Two- Dimensional Ultrasonic Imaging
}

\author{
Yanguo Hu, Yaying Zhao,Dean Fu \\ Department of Imaging, Second Affiliated Hospital of Dalian Medical University, Liaoning, China
}

\begin{abstract}
In the development of traditional Chinese medicine in China for five thousand years, the tongue clinic occupies a very important position. In Chinese medicine, it is an irreplaceable role in the diagnosis and treatment of Chinese medicine of important method. Tongue diagnosis as one of the most characteristic of Chinese medicine diagnosis has been from the previous subjective observation, relying on the experience to judge the era into the new era of tongue diagnosis objectification, quantification, and the introduction of computer digital image processing technology to more Accurate diagnosis. This article will mainly introduce the current in the Chinese and Western medicine on all aspects of the tongue diagnosis of the objective, micro, quantitative progress, the characteristics of tongue images and a variety of processing techniques, including tongue image automatic segmentation, and tongue fat and thin The Relationship and Modernization of Organ Pathology in Viscera. At the same time, a tongue-sized measurement method is introduced. Two-dimensional ultrasound imaging is used as a means of inspection to establish a set of ultrasonic tongue-sized measurement methods. The measured parameters include the two longitudinal planes of the tongue and the crosssection of the tongue. Out of the tongue size of the data, the overall analysis of the size of the tongue shape. This method combines some of the configuration line spacing, area, and circumference measurement functions to directly measure the data. Finally, through the accuracy test of the measurement method, it is proved that the measurement result of tongue two-dimensional ultrasound imaging is accurate. All the efforts of modernization are committed to the Chinese medicine tongue medicine to scientific can be quantified; Chinese medicine tongue diagnosis will also make a greater contribution to modern medicine.
\end{abstract}

KEYWORDS: Tongue diagnosis objective; Tongue image segmentation; Tongue body fat thin; Two-dimensional ultrasound imaging

\section{Introduction}

\subsection{Overview}

Tongue diagnosis, is fully embodies the characteristics of traditional Chinese medicine diagnosis of one of the methods, with a complete, systematic, rigorous theoretical system, for a long time, in the clinical diagnosis of Chinese medicine play a huge role. To better inherit the experience of the ancients' tongue diagnosis, to speed up the development of tongue diagnosis theory, so that tongue diagnosis better service in modern Chinese medicine, it must be extensive, deep, active research, and the existing research Ideas, methods carefully combed, has been in the study of the experience and results, to seriously sum up. Can the tongue diagnosis really objective, micro-quantitative, and quantitative.

Traditional Chinese medicine is on the basis of experience on the subjective understanding of the tongue diagnosis, the results will inevitably be biased, and modern medicine to the introduction of computer technology in the tongue of traditional Chinese medicine, let the data, objective at the same time, to be dissected Learning analysis of the traditional Chinese medicine tongue diagnosis of the image analysis proposed a series of automatic segmentation method, but also for the analysis of tongue diagnosis to provide a more convenient and convenient and efficient technical support.

First of all, the traditional research methods combined with modern medical methods such as electron microscopy, thermal imager, B ultrasound, tongue and other widely used instrument, laboratory hemorheology detection, tongue coating off cell detection and some molecular biotechnology applications. So tongue research is brought into a new brand new platform.

Secondly, the development and application of electronic computer technology and network information technology have realized the omni-directional extraction of tongue image information and the automatic analysis of tongue color,

Copyright (C) 2017 -. This is an Open Access article distributed under the terms of the Creative Commons Attribution-NonCommercial 4.0 International License (http://creativecommons.org/licenses/by-nc/4.0/), permitting all non-commercial use, distribution, and reproduction in any medium, provided the original work is properly cited. 
moss color, thick fur thickness, crack and moistening and so on. This has entered a modern, objective, information, standardization of the rapid development of the road.

Tongue diagnosis in the theory of traditional Chinese medicine system and the practice of clinical treatment occupies a pivotal position. It is an important means to inherit the traditional Chinese medicine diagnosis by integrating the computer technology and image processing technology with the traditional Chinese medicine tongue diagnosis to realize the quantitative, objective and standardization of the tongue diagnosis, so as to realize the modernization of the traditional Chinese medicine tongue diagnosis.

\subsection{Advances in Objectivity Research}

\section{Tongue, tongue, and other objective study of tongue diagnosis}

Tongue is a very sensitive indicator of the changes in the body of the body, can objectively reflect the integrity of the people, the nature of evil, the disease advance and retreat, the exact dialectical prevention, to determine the outcome of the disease, to guide the prescription medication has important significance.

In the study of the objectification of the tongue, the Beijing University of Technology first proposed three methods. The first method is to use a variety of support vector machine learning algorithm on the tongue to do qualitative, quantitative description. The second method is based on the learning vector quantization of the artificial network of moss color for automatic classification method. In the design of classifier, the method of sample screening based on 2R0 criterion is proposed, and the Fisher ratio is used as the basis of spatial selection of chromaticity, which effectively improves the accuracy of classification. The third method is to supervise the FCM clustering algorithm to apply the concept of fuzzy point, design multi-layer to the fuzzy processing and tongue type calibration method, can be effective on the color of the tongue coating.

In the aspect of tongue, Wang et al. used a multi-scale edge detection method based on Vector Prewitt operator to quantitatively analyze the crack characteristics of the tongue, and obtain a quantified crack index to reflect the number and depth of the crack.

In the objective study of the tongue diagnosis, Cai et al. have not been able to meet the problem of collecting the tongue image of the sleeping tongue patient, and put forward a new method of collecting the device of the tongue image analyzer. Using a digital camera comes with flash as the instrument light source, reducing the size of the instrument to improve its electrical safety; the use of integrating sphere lighting geometric conditions, efforts to improve the uniformity of light. Cai et al. also carried out experimental research on the new collection device, and to determine the collection parameters. For the change of the color of the tongue image caused by the change of the optical imaging environment of the new device, the color conversion of the image from the optical environment of the new tongue collection device to the standard environment is realized by using the calibration color plate and the correction algorithm of the off-line color. This is to achieve the tongue of the indicators analysis.

In recent years, there have been a lot of long-term, multi-level, multi-disciplinary cross-cutting excellent features, but there are still many problems to be solved. Such as the comprehensive identification of the tongue in the comprehensive identification, analysis and processing there is a clear lack of information on the human tongue and clinical interpretation of the application, has not fully and clinical combination of traditional Chinese medicine, making the tongue like the lack of clinical information Sexual, not conducive to clinical promotion; multidisciplinary integration level still needs to be strengthened. In the future, we will under the guidance of traditional Chinese medicine theory, closely with clinical practice, to carry out the organization of various disciplines of experts in close cooperation, the use of computer vision systems, optical technology and image analysis technology and other clinical modern science and technology, Objective and unified identification standards for the standardization of Chinese medicine diagnosis, quantitative and accurate to provide an objective basis to promote the development of Chinese medicine academic.

\section{Application and progress of computer technology in tongue diagnosis}

Since the late 1950s, a small number of scholars in the fluorescent staining, tongue microcirculation, scraping tongue smear, animal experiments and other aspects of a lot of research work, and achieved certain results for the modern study of tongue diagnosis has laid a solid foundation The In 1986, China's first computer image recognition technology used in the study of tongue diagnosis, 'traditional Chinese medicine moss spectrum' on the representative of the color image of the tongue modeled, dealing with normal, cold, real heat, The results showed that the color of the tongue and the tongue of the normal people were different in red, green and blue.

This is the application of computer technology and image processing in the modern research of tongue diagnosis, which shows that it is feasible to locate and quantify the tongue image by using computer image recognition technology. Since then in this area of technology applications will gradually emerge. China's researchers also slowly have more technical ideas can be regarded as tongue diagnosis in computer technology theoretical basis: 
Sun's vision of the standard light source conditions, the application of a new type of color camera intake of tongue image, after computer processing for digital images (quantitative analysis), and then refer to the main organs of different syndromes and normal, and then classified in the Chinese medicine experts The paper summarizes the objective basis of the quantitative analysis of the tongue diagnosis, and establishes a recognition system which focuses on the change of the chromaticity of the tongue. Guo Zhenqiu and others put forward the microcosmic technology to be combined with computer technology, applied to the study of the objective study of tongue diagnosis. Guo's idea is to use the microscope to observe the tongue nipple accurately microscopic observation, microscopic measurement, and then the microscopic image processing, and finally analyze the micro-tongue papilla and the relationship between the disease syndromes, and strive to find the tongue Objective indicators.

The above two studies, the two have their own focus, Sun is mainly from the perspective of macro-image, and Kwok from the micro-point of view, can be said to have their own strengths, have opened up a new study for the tongue diagnosis. However, the computer technology is a solid theoretical basis in the application in the field of tongue diagnosis.

After the basic theoretical assumptions, people began to explore the computer tongue recognition system and its application software design. Zhao et al. designed the 'tongue, tongue coating quantitative analysis system.' The system uses a linear array camera and digital circuit, respectively, through the red, green and blue color filters, the tongue as a slide to shoot, and digital, enter the computer for storage and processing; in the collection, the data Calibration is very necessary, with three-color relative value to statistics and analysis, the initial identification of a variety of tongue and tongue classification parameters, according to classification parameters for classification calculation.

In this theory, Kui et al. believe that the color of the tongue by the tongue mushroom-like nipple distribution density and each mushroom-shaped papillary blood vessel loop distribution area and the color of the blood vessels to determine the decision, borrowed the computer image processing and graphics amplification technology, the common digestive system of the tongue was observed. The method is briefly described as follows: first through the connected highresolution color camera and microscope, the tongue to enlarge to 10 times in the patient's tongue and left and right side of the tongue to take a diameter of $0.5 \mathrm{~cm}$ circular area, record The number of mushroom-shaped nipples, and find the density of the mushroom-shaped nipple distribution; and then from any of the 10 mushroom-shaped nipples, 130 times the magnification of each mushroom-shaped papilla of the vascular loop and then the computer image scan and the average value of the three primary colors of R, G and B is finally taken as the distribution density of the mushroomshaped papilla, and the result is the result of the detection.

The tongue diagnosis system of the above-mentioned various computers can accurately identify the tongue image to a certain extent, and provide reference information for clinical medicine, which shows that the application of computer in the field of tongue diagnosis can make the system reach, Even more than the traditional tongue diagnosis effect.

\section{Characteristics of tongue image and the main tongue image segmentation method}

\subsection{The shape and structure of the tongue}

The shape of the tongue is divided into upper and lower surfaces; the above circular round called the tongue, the tongue on the back by the chevron of the boundary groove will be divided into the tongue in front of the tongue, the rear side of the tongue for the tongue. There is a tongue in the middle of the tongue is a mucosal folds. Tongue belt on both sides of the lower side of a small uplift, known as the sublingual $\mathrm{Fu}$, there are submandibular gland duct, sublingual common opening. In the sublingual Fu after the lateral, there is a mucosal folds, and the tongue next to the fold, the deep sublingual gland.

The structure of the tongue from the surface covered with a mucous membrane, with tongue and muscle composition, tissue structure can be divided into three layers, respectively, mucosal layer, and Lamina propria and muscular. The mucosal layer is usually divided into four layers, from shallow to deep: keratinized layer, granular layer, spine cell layer, basal layer; due to the structural characteristics of the mucosa layer, determines its active metabolism, cell renewal faster, about every three Day is better once, one of the most active places for oxidative metabolism in the body. And the Lamina Propria with connective tissue, containing blood vessels, lymphatic vessels, nerves and tongue glands, etc., can see a small amount of lymphocytes in the tongue layer of the inherent layer of the formation of many sizes ranging from dermal papilla, Into the mucosal epithelium. The most muscular layer is located in the inherent layer, there are skeletal muscle composition of muscle bundle, muscle bundle between a small amount of connective tissue, during which visible blood vessels and nerves. 


\subsection{Tongue color, tongue shape and pathology, tongue texture characteristics analysis}

\section{Character analysis of tongue}

Tongue characteristics are including tongue and tongue in the performance of various circumstances, is the main basis for TCM syndrome differentiation. The body's tongue can be described in terms of color, gloss and shape, and the tongue can be described by its color and texture. Tongue color and pink, pale, red and purple; tongue and fat, thin, crack and thorns of the points; moss color white, yellow, gray, black, blue, brown of the other; , There are thin, thick, run, dry, rot, tired of the points; In addition, the human tongue there are soft, hard, shrink, tremble and other dynamic features. According to these characteristics, we can use the method of feature extraction of tongue and tongue on the objective quantitative analysis.

\section{Tongue, shape characteristics and pathology}

The previous section has been introduced, the tongue, tongue characteristics of the analysis, this section will mainly introduce the tongue, and tongue-shaped characteristics of the tongue color, that is, the color of the tongue, generally divided into pink, Red, purple, bruising five kinds of tongue color; these five colors represent different clinical significance, light red that blood to reconcile, means full of effort, strong stomach; pale white blood deficiency, yang, white tongue The main tongue is hot, Yin; hot tongue main heat hyperactivity, Yin Huo-wang, generally evolved from the red tongue evolved; green tongue is divided into light purple tongue, purple tongue, purple tongue, Bluish spot tongue, the main blood gas is poor; the following is the case of the tongue pattern:

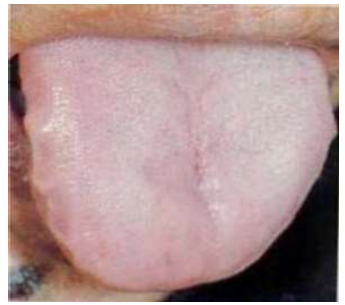

Figure 1 Pale White Tongue

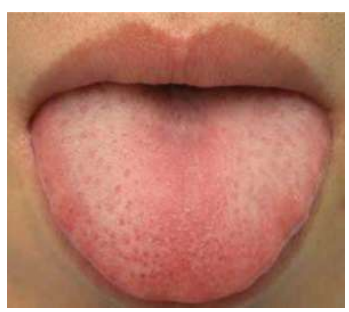

Figure 2 Tongue

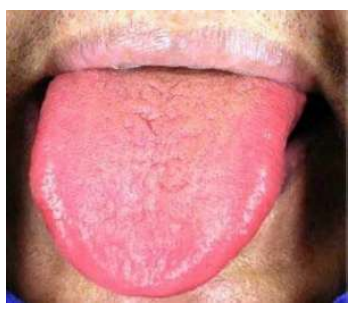

Figure 3 Green Tongue 


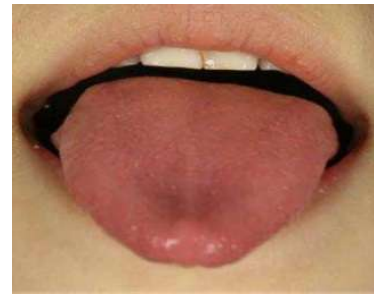

Figure 4 Red Tongue

\section{Analysis of tongue texture characteristics}

In this paper, we use the multi-scale edge detection method based on vector Prewitt operator to analyze the tongue crack characteristics quantitatively. In this paper, we analyze the characteristics of tongue texture, which includes texture analysis of tongue crack and tongue greasy. For the former, Wang et al. Get a quantified crack index, and to reflect the number and depth of the pattern. While the latter is the defenders and other people according to the characteristics of greasy moss texture, through the improved subspace method to distinguish the tongue layer into a fixed size of the block, the projection length ratio of each block as its classification and identification characteristics, in order to analyze it's The density of the texture structure is classified when the credibility is not high enough, combined with the texture roughness characteristics of the expressed particle thickness. On the basis of the classification results, the Annihive index and graph description of the whole tongue image are given.

\subsection{Automatic tongue image segmentation method}

\section{Introduction of segmentation method of effect deviation in tongue segmentation}

Edge-based segmentation method which is first detects the discontinuity or mutability of local characteristics in the image, and then connects them into boundaries, which in turn divide the image into different areas. The difficulty of this method lies in the edge detection will produce anti-noise and detection of the contradiction between the accuracy. If the detection accuracy is improved, the pseudo edge of the noise will lead to unreasonable contours. If the noise is improved, the deviation of the contours and the position will be generated. Therefore, the world is often used in this regard is based on the pixel attribute edge detection method, based on the mathematical morphology of the edge detection method and based on the parameter deformation template edge detection method.

The first is based on the pixel attribute edge detection method, which is the most common method of edge detection, is used to detect the brightness of the discontinuity. This method is mainly to consider the pixel data, and does not take into account the actual division of the object in the distribution of pixels and the characteristics of the actual target, starting from the point of the border, according to the grayscale gradient and other information to find similar to other border points, Will eventually form a closed tongue border, so that you can determine the part of the inside is the tongue, the advantage of this method is the edge of the positioning accuracy, the operation of the fast. The first-order detection operator of the first-order Roberts, Prewitt, and Canny and other second-order operators are tried and compared, and then they can find their common defects: $\square$ It is difficult to get a complete closed contour; The body of the cracks and the tongue around the teeth, collar and other objects with strong boundary characteristics of the object will also be easy to affect the effect of segmentation; $\square$ Some tongue color and skin color close to the patient's case picture on the tongue and lips border will Fuzzy is not easy to distinguish. In general, the traditional edge detection operator on the image containing noise on the treatment effect is not ideal, the loss of the effective edge, cannot be effective to suppress noise. Therefore, it is necessary to add a large number of human intervention after the boundary detection, to assist in the deletion of the detected boundary, and then to add the missing boundary in order to form a complete contour of the tongue, and finally to be divided.

\section{Region-based segmentation method commonly used in tongue image segmentation}

The region-based general segmentation method applied to the segmentation of the tongue image has a threshold method and a region growing method. Threshold method is a simple and effective method of image segmentation, the basic theory is to use one or more thresholds of the gray level of the image is divided into several levels, gray value in the same pixel is the same target the advantage of the threshold segmentation method is that the calculation is simple, the operation is more efficient and fast, and it is suitable for the case where the target has a strong contrast to the background gray scale. More importantly, the background or the gray scale of the object is relatively simple the boundary of the closed and connected area can always be obtained. When a tongue image satisfying such a condition is obtained, its grayscale histogram can be viewed as a mixture of two unimodal histograms corresponding to the tongue and the background, respectively. If the size of the two distributions is close to the mean and the distance is sufficiently 
far and the mean square error is small enough, the histogram is bimodal and the dynamic threshold is easier to select to segment. However, in the actual treatment to meet these conditions, the tongue image is very small, most of the tongue inside the tongue because of the existence of the tongue, so that the tongue of the histogram is difficult to show a single peak distribution. Moreover, some of the tongue of the tongue and the lips, skin and other colors close to this method will be difficult to accurately locate the border. In this regard, Ren and others for the characteristics of color tongue image, taking into account the characteristics of several commonly used color image segmentation method, proposed a gray space based on the automatic threshold of the selected segmentation algorithm, and its application in the color of the tongue image segmentation. Yang et al. reported that the traditional threshold method in the extraction of the tongue contour defects, the collected images are first balanced with the histogram, the pretreatment, so as to improve the image color resolution, and then according to the tongue shape, location and other a priori information, with geometric methods to modify the mouth and lips affected by the edge, the final completion of the extraction of tongue work.

\section{Analysis of tongue and body fat and thin}

\subsection{The relationship between tongue and body fat and body organs and organs}

The fat and thin tongue is one of the important bases of TCM dialectical disease. The size of the body of the tongue reflects the health of the body organs or not, in the traditional Chinese medicine, the tongue fat and thin diagnosis classification is very simple, divided into fat big tongue and thin tongue; which fat big tongue 'tongue color pale, tongue Body fat tender, bigger and thicker than normal tongue, and even full of mouth, 'the main spleen and kidney yang, gasification disorders, water within the stop. Tongue body thin and thin than the normal tongue, known as the 'thin tongue', the main qi and blood deficiency and Yin and Blood.

Tongue through the meridians and the five internal organs connected, so the human body organs, blood, body fluid of the actual situation, the severity of the disease changes, may be objectively reflected in the tongue, the Chinese medicine will be divided into tongue, tongue, tongue and tongue side, That the tongue is a heart and lung, the tongue is the kidney, tongue on both sides of the liver and gallbladder, according to different parts of the different parts of the viscera, but cannot be mechanical to see, to combine other symptoms and signs, and scientific instruments to be reasonable The correct result.

\subsection{A measure of the size of the tongue: two-dimensional ultrasound imaging}

\section{Materials and measurement methods}

The experimental equipment is a DIASONICS-SPECT RA-PLUS color Doppler device, the measurement and analysis method is: the subjects take pillow supine position, head back to the neck angle for the right angle or obtuse angle, in order to facilitate the ultrasonic probe Submandibular area placed. The B-type ultrasound imaging diagnostic instrument is for the measurement of tongue size. The choice of level at the time of measurement is the cross section of the tongue and the longitudinal section of the tongue:

First, the tongue cross-section: adjust the PRF (high frequency repetition rate) value to 1000 , and $3.5 \mathrm{MHz}$ probe, transverse line placed in the human submandibular area, so that the probe long axis and chin to the neck point of connection perpendicular to each other. And then slowly move the probe from front to back until the side or both sides of the tongue deep began to appear, and then adjust the probe in this position to detect the direction to make it consistent with the subject supine plane, and then fixed the probe. 5 seconds after swallowing and then extract the time of the tongue cross-sectional image, and as a measurement image (Figure 5)

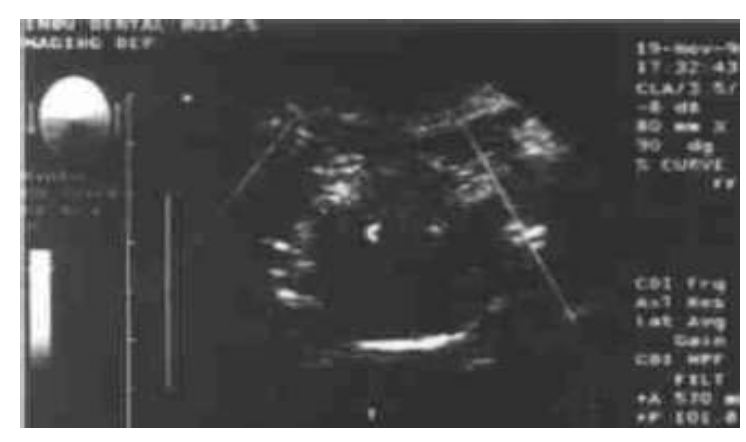

Figure 5 Tongue cross-sectional image 
Second, the tongue part of the longitudinal section: the $3.5 \mathrm{MHz}$ probe longitudinal placed in the middle of the lower jaw area, so that the long axis of the probe and the chin point to the neck line parallel to each other, and keep the direction of the head of the detection direction fixed. Adjust the position of the probe before and after the tongue and tongue after the echo-free sound of the front can be displayed on the display, and then we fixed the probe at this location. In the $5 \mathrm{~s}$ after swallowing, the longitudinal image of the tongue is extracted at this moment as the measurement image (Figure 6).

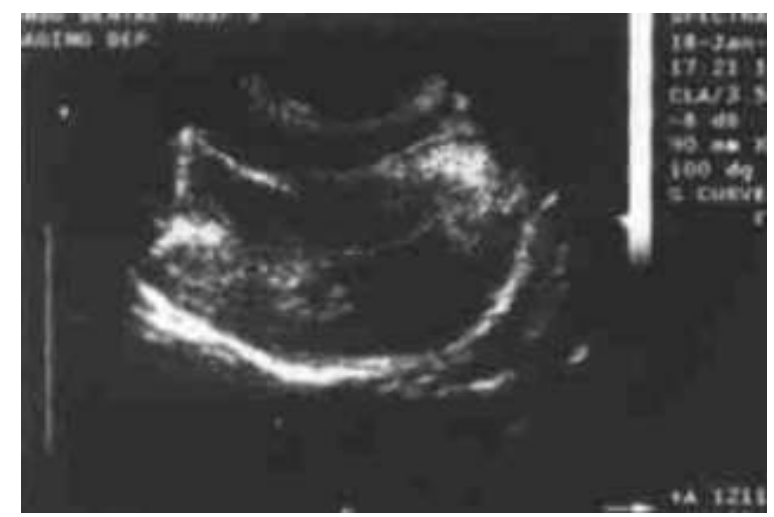

Figure 6 Center of the middle section of the image

Selection and measurement method of measurement of tongue size: The measurement of the following items is made using the measurement function of line distance, area and circumference configured by the ultrasonic imaging diagnostic apparatus. A. Tongue diameter is long: the cross section of the cross section of the image on the widest width is the width of the tongue lateral. B. Perimeter and area of the cross section of the tongue: represents the overall size of the cross section of the tongue. C. The total length of the tongue: the distance from the tip of the tongue to the last point of the tongue, representing the longitudinal length of the tongue. D. The length of the tongue front: the distance from the tongue point to the tongue band, here represents the length of the tongue that is free from the tongue anterior part of the tongue. In the tongue band at the Genioglossus and tongue muscle are attached to the forefront. E. The area and circumference of the longitudinal section of the tongue: represents the overall size of the tongue sagittal section. F. Tongue in the longitudinal section of the tongue on the diameter of the tongue: this length from the tongue, along the tongue back to the tongue until the end of the tongue. This should be an indicator of the length of the tongue, regardless of the tongue function. The above measurement parameters can be reflected from different angles to the shape and size of the tongue, a comprehensive analysis can see the overall size of the tongue.

\section{Accuracy test of experimental results}

Measurement of the accuracy of the measurement results: We use the tongue-sized magnetic resonance imaging measurement methods, and we established the tongue-sized ultrasound measurement method, respectively, the median longitudinal image of the tongue to measure the magnetic resonance measurements of Standard, compare the accuracy of tongue - sized ultrasound measurements. A total of 10 subjects (numbered 1-10) with no metal restorations were randomly selected. The method of measuring the size of the tongue in the middle longitudinal section of the tongue and the measurement items were measured by magnetic resonance method and ultrasonic method respectively. The results showed no significant difference between these measurements $(P>0.05)$ (see Table 1). Therefore, with the measurement of magnetic resonance as the standard, we believe that the results of tongue-sized ultrasound imaging measurements are accurate. 
Figure 1: Verification of the accuracy of the project in the middle of the tongue

\begin{tabular}{|c|c|c|c|c|c|c|c|c|c|c|}
\hline \multirow[t]{2}{*}{ Subjects } & \multicolumn{2}{|c|}{$\begin{array}{l}\text { Length of } \\
\text { Tongue }\end{array}$} & \multicolumn{2}{|c|}{$\begin{array}{l}\text { Tongue } \\
\text { Front } \\
\text { Length }\end{array}$} & \multicolumn{2}{|c|}{$\begin{array}{l}\text { Tongue Apex } \\
\text { Long }\end{array}$} & \multicolumn{2}{|c|}{$\begin{array}{l}\text { Tongue } \\
\text { Longitudinal } \\
\text { Section } \\
\text { Circumference }\end{array}$} & \multicolumn{2}{|c|}{$\begin{array}{l}\text { Tongue } \\
\text { Longitudinal } \\
\text { Section Area }\end{array}$} \\
\hline & $\begin{array}{l}\text { Ultr } \\
\text { ason } \\
\text { ic } \\
\text { Mea } \\
\text { sure } \\
\text { ment } \\
\text { s }\end{array}$ & $\begin{array}{l}\text { Magne } \\
\text { tic } \\
\text { Resona } \\
\text { nce } \\
\text { Measur } \\
\text { ements }\end{array}$ & $\begin{array}{l}\text { Ultr } \\
\text { ason } \\
\text { ic } \\
\text { Mea } \\
\text { sure } \\
\text { ment } \\
\text { s }\end{array}$ & $\begin{array}{l}\text { Mag } \\
\text { netic } \\
\text { Reso } \\
\text { nanc } \\
\text { e } \\
\text { Mea } \\
\text { sure } \\
\text { ment } \\
\text { s }\end{array}$ & $\begin{array}{l}\text { Ultra } \\
\text { sonic } \\
\text { Meas } \\
\text { urem } \\
\text { ents }\end{array}$ & $\begin{array}{l}\text { Ultra } \\
\text { sonic } \\
\text { Meas } \\
\text { urem } \\
\text { ents }\end{array}$ & $\begin{array}{l}\text { Ultras } \\
\text { onic } \\
\text { Meas } \\
\text { ureme } \\
\text { nts }\end{array}$ & $\begin{array}{l}\text { Ultraso } \\
\text { nic } \\
\text { Measu } \\
\text { rement } \\
\mathrm{s}\end{array}$ & $\begin{array}{l}\text { Ultra } \\
\text { sonic } \\
\text { Meas } \\
\text { urem } \\
\text { ents }\end{array}$ & $\begin{array}{l}\text { Ultra } \\
\text { soni } \\
\text { c } \\
\text { Mea } \\
\text { sure } \\
\text { ment } \\
\text { s }\end{array}$ \\
\hline 1 & 53.2 & 54.9 & 31.2 & 33.1 & 92.4 & 93.7 & 163.1 & 159.7 & 1550 & 1478 \\
\hline 2 & 62.3 & 64.7 & 33.5 & 32.9 & 94.7 & 91.3 & 168.3 & 170.4 & 1273 & 1324 \\
\hline 3 & 48.9 & 51.4 & 28.4 & 26.7 & 86.9 & 84.5 & 140.1 & 138.6 & 1231 & 1265 \\
\hline 4 & 57.1 & 55.4 & 34.3 & 35.9 & 91.5 & 95.4 & 164.8 & 161.3 & 1323 & 1287 \\
\hline 5 & 64.8 & 66.4 & 30.8 & 31.9 & 93.7 & 94.8 & 158.9 & 162.8 & 1172 & 1113 \\
\hline 6 & 61.3 & 58.4 & 33.7 & 35.2 & 95.6 & 93.1 & 160.3 & 163.5 & 1211 & 1175 \\
\hline 7 & 71.9 & 67.4 & 36.7 & 38.4 & 109.8 & 112.4 & 177.2 & 179.4 & 1353 & 1296 \\
\hline 8 & 55.4 & 53.1 & 29.4 & 30.7 & 96.2 & 93.6 & 167.8 & 164.2 & 908 & 1037 \\
\hline 9 & 64.6 & 67.3 & 34.5 & 35.6 & 98.7 & 96.8 & 171.2 & 167.1 & 1302 & 1254 \\
\hline 10 & 58.4 & 59.7 & 33.2 & 31.8 & 105.3 & 106.7 & 158.9 & 163.7 & 1143 & 1228 \\
\hline P值 & & 0.05 & & .05 & & & & 05 & & 05 \\
\hline
\end{tabular}

\section{Discussion}

The measurement of tongue size has always been a problem, mainly because the back of the tongue has been extended to the pharynx, most of its bottom is attached to the bottom of the mouth, and it is difficult to directly observe the measurement. The detachment of the tongue root and the scalability of the tongue itself make it difficult to determine the state of the measurement and to increase the difficulty of the measurement. For decades, people have been trying to measure the size of the tongue, from the original to take the tongue of the impression measurement method until the past few decades began to use imaging methods to measure, such as CT imaging measurement, X-ray Bit positioning film measurement method, and three-dimensional ultrasonic measurement method. These methods have some limitations in the application of orthodontic clinical examinations, such as when measured in a method of taking a tongue-like impression, the state of the tongue is subjectively controlled by the subject and does not May be extracted to include the root of the root of the impression, it cannot be the whole tongue measurement; in the skull lateral positioning chip cannot distinguish between the tongue muscle and extracorporeal muscle boundaries, and even outside the tongue of the boundaries cannot be fully revealed, can only use some bone markers to calibrate the boundary line of the tongue, not the true tongue of the border line; another CT, MRI and three-dimensional ultrasound means to clearly identify the boundaries of the tongue, but its high check the cost so that these methods cannot be routine orthodontic clinical examination. So its clinical significance is not, has not been used for clinical medical treatment.

Now we need for orthodontic clinical examination, with two-dimensional ultrasound imaging as a means of inspection, the establishment of a set of ultrasonic tongue size measurement method, the measurement parameters, including the tongue longitudinal section and the tongue cross-section of the two measured plane, reflecting The tongue size of the data, the overall analysis of the size of the tongue shape. Through the accuracy and repeatability test of the measurement method, it is proved that the measurement method of the tongue two-dimensional ultrasonic imaging method is accurate and reproducible. This method of ultrasound is harmless to the human body, and the cost of inspection is low, easy to promote the application, can be used as a tongue size measurement and analysis means.

Before the analysis, we used the tongue segmentation method to extract the contours of the tongue, and then according to the characteristics of the tongue body tongue and fat automatic analysis. First calculate the aspect ratio of the tongue, the tip of the tongue to the tongue as the distance between the tongues, the distance between the two tongue sides called the width of the tongue. Thin tongue aspect ratio is generally larger, and fat big tongue aspect ratio is generally smaller. Second, the shape of the tongue and tongue fat and thin there is a certain 
relationship. In general, thin tongue tends to be sharper, and fat tongue is more blunt tongue. We can these two characteristics with the image language to express, and then you can automatically analyze the body fat and thin. To make an edge with a contour line parallel to the axis of the tongue, the parallel length parallel to the central axis corresponds to the length of the tongue and the length of the side perpendicular to the central axis. The aspect ratio of the tongues can be approximated by the aspect ratio of the rectangle. According to the above method, we can measure the size of the tongue, and then compared with the standard tongue; you can determine the body fat and thin, so as to prevent the disease, to achieve good clinical results.

\section{Conclusions}

The research work of this paper discusses the problem of tongue image and tongue fat and thin, and lists the current process and problems of tongue diagnosis. The tongue diagnosis is one of the important contents of traditional Chinese medicine diagnosis and treatment. It is gradually formed with the development of Chinese medicine a unique and important diagnostic method, almost every routine examination of traditional Chinese medicine. Over the past half century, with the progress of human science, the study of tongue diagnosis has made great achievements, we are in the traditional theory of continuous inheritance and innovation, the tongue diagnosis of the relevant research systematization; at the same time the scope of the study The depth of the study also reached the level of cells, subcellular cells and molecules; research methods are increasingly diversified, advanced; tongue diagnosis of the relevant instruments are also gradually Of the mature, tongue diagnosis of animal experiments began to sprout and so on. But we see the results at the same time have to see this one of the shortcomings: First, the tongue, surface image information collection, the equipment has not yet established to achieve the industry and national standards. Image acquisition device storage format is the lighting environment between the larger differences, poor compatibility with each other. Each research unit uses its own internal standards, hindering the exchange of technology and widespread use. Secondly, the research on the tongue reading information carried out by domestic and foreign Chinese medicine practitioners focuses on the quantitative research of the image itself, and has not yet established the method of effective quantification (or classification). Therefore, the tongue, face diagnosis of information on the degree of clinical acceptance has also been greatly affected. Therefore, this paper argues that the performance of information collection equipment should be improved, the standard method should be implemented, the standard body model should be constructed and the relevant technical standards should be formulated. At the same time how to follow the premise of traditional Chinese medicine under the premise of improving the traditional Chinese medicine tongue, face diagnosis of the scientific basis and content of the study, to promote standardization and standardization of the process, is also an important subject of traditional Chinese medicine diagnosis. We also have to strengthen the tongue of Chinese medicine, objective research, to promote the relevant research equipment in clinical, scientific research in a wide range of applications. Only in this way can we better inherit and carry forward our treasures of Chinese medicine.

\section{References}

1. Journal of Lanzhou Jiaotong University, 2006,25 (6): 89

2. Zhao Zhongxu, Wang Aimin, Shen Lansun.Color tongue image segmentation based on mathematical morphology and HIS model [J]. Journal of Beijing University of Technology, 1999, 25 (2): 67.

3. FU Zhi-cheng, LI Xiao-qiang, LI Fu-feng. Photot image segmentation based on radial edge detection and Snake model [J] .Journal of Image and Graphics, 2009,14 (4): 688.

4. Ren Jijun. Color image segmentation algorithm based on automatic threshold selection of gray space [J]. Journal of Shaanxi University of Science and Technology, 2005, 23 (2): 73.

5. Yang Dasheng, Chen Hehui, Zou Fengmei, et al. An effective automatic segmentation algorithm for tongue body [J] .Application Research of Computers, 2007,24 (2): 170-172.

6. Sun Yang, Luo Yu, Zhou Changle. A Segmentation Algorithm for Chinese Tongue Image Based on Split-merge Method and Its Realization [J] .Journal of Image and Graphics, 2003, 8 (12): 1397-139.

7. MA Chao, TANG Zhi-de, TANG Lin. Application of image segmentation technique in tongue diagnosis of traditional Chinese medicine [J]. Computer Simulation, 2008, 25 (2): 215-218.

8. Li Naimin, China Tongue Daquan [M]. Beijing: Xueyuan Publishing House, 1995: 20-21.

9. Chen Zelin, Chen Meifang. Tongue study [M]. 5th edition. Shanghai: Shanghai Science and Technology Press, 1982: 71-74.

10. Chen Zelin. Pale red, pale white, red drop, bruising four kinds of tongue tongue microcirculation research. Shanghai Chinese Medicine Journal, 1995: 21-58.

11. Chen Zhangrong. Tongue value of clinical observation of 566. Fujian Traditional Chinese Medicine, 1998: 58-66.

12. Brodie AG. Behavior of normal and abnormal facial growth pat-terns. Am J Oral Surg, 1947, 27: 633 